\title{
Volatility clustering and scaling for financial time series due to attractor bubbling
}

\author{
A. Krawiecki ${ }^{a, b}$, J. A. Hołyst ${ }^{a, b}$, and D. Helbing ${ }^{b}$ \\ ${ }^{a}$ Faculty of Physics, Warsaw University of Technology, Koszykowa 75, PL-00-662 Warsaw, \\ Poland \\ ${ }^{b}$ Institute for Economics and Traffic, Dresden University of Technology, D-01062 Dresden, \\ Germany
}

\begin{abstract}
A microscopic model of financial markets is considered, consisting of many interacting agents (spins) with global coupling and discrete-time thermal bath dynamics, similar to random Ising systems. The interactions between agents change randomly in time. In the thermodynamic limit the obtained time series of price returns show chaotic bursts resulting from the emergence of attractor bubbling or on-off intermittency, resembling the empirical financial time series with volatility clustering. For a proper choice of the model parameters the probability distributions of returns exhibit power-law tails with scaling exponents close to the empirical ones.
\end{abstract}

PACS numbers: 89.90.+n, 05.40.-a, 05.45.-a

Key words: On-off intermittency, attractor bubbling, stock market dynamics, power-law scaling, fluctuating social interactions. 
In recent years, both the analysis and modeling of financial time series have attracted a growing interest in statistical physics [1-11], motivated by a quest for a possibly universal dynamics underlying different markets. Indeed, various financial time series share similar properties, e.g., relative price changes (returns) show intermittent occurrence of large bursts (volatility clustering), resulting in the power-law scaling of tails in their probability distributions, as well as of the autocorrelation function of their absolute values [3]. Large-scale microscopic simulations and analytic studies [4-11] revealed that such scaling can result from phenomena as diverse as, e.g., percolation [4] or stochastic processes with multiplicative noise [8,9,12]. It was also shown [5, [7, 9, 10] that in some models of market dynamics volatility clustering resembles on-off intermittency, an extreme kind of intermittency characterized by a sequence of large chaotic bursts separated by almost quiescent laminar phases [13], or attractor bubbling, the predecessor of on-off intermittency in the presence of additive noise [14].

In this Letter we present simulations of financial time series based on a microscopic model of many interacting agents with discrete-time dynamics. The agents are treated as spins exposed to fluctuations (a "heat bath") and coupled by randomly time-dependent Ising-like interactions. Related spin models, but with time-independent interactions, have been proposed for economic [11] and social systems, where they are theoretically and empirically supported by the social impact theory of opinion formation [15]. In contrast, we emphasize that the social interactions and the communication network among individuals are time dependent. They are also more and more determined by different kinds of long-range communication instead of spatial neighborhood which makes the topological structure of the interaction network unimportant. We show that, if the average strength of these interactions (which reflect the average reaction of agents to price changes) varies randomly in time, the system displays volatility clustering, and probability distributions of returns resemble those of empirical financial time series. A peculiar feature of our model is that the dynamics on a microscopic level is stochastic, but interactions between large numbers of agents cause the macroscopic dynamics to become typical of dynamical systems with attractor bubbling. In particular, in the mean-field approximation our model reduces to a generic model for attractor bubbling. Another important feature is the presence of various sources of stochasticity in the microscopic dynamics. First, the agents make decisions (on investments) under the influence of the external environment and other agents. The variation of the strength 
of this influence is simulated by explicit random updates of the interaction strengths with the environment and among agents at every time step. Besides, the final agent decision is determined according to a probabilistic rule that mimics the the uncertainty in human decision-making.

Explicitly, we consider $i=1,2, \ldots N$ agents (spins) with orientations $\sigma_{i}(t)= \pm 1$, corresponding to the decision to sell $(-1)$ or to buy $(+1)$ a share of a traded stock or commodity at discrete time steps $t$. The orientation of agent $i$ at time $t+1$ depends on the local field

$$
I_{i}(t)=\frac{1}{N} \sum_{j=1}^{N} A_{i j}(t) \sigma_{j}(t)+h_{i}(t),
$$

where $A_{i j}(t)$ are time-dependent interaction strengths among agents (reflecting their social or communication network), and $h_{i}(t)$ is an external field reflecting the effect of environment (e.g., access to external information which can differ between agents). The interaction strengths and external fields change randomly in time,

$$
\begin{aligned}
A_{i j}(t) & =A \xi(t)+a \eta_{i j}(t), \\
h_{i}(t) & =h \zeta_{i}(t)
\end{aligned}
$$

where, for simplicity, we assume that $\xi(t), \eta_{i j}(t)$, and $\zeta_{i}(t)$ are random variables with no correlation in space and time, uniformly distributed in the interval $(-1,1)$. According to Eq. (2), $A$ is a measure of the randomly varying average interaction strength between agents, $a$ is a measure of the random deviations of the individual interaction strengths $A_{i j}(t)$ from the average, and $h$ is a measure of the random influence of the environment. The strengths $A_{i j}(t)$ can assume both positive and negative values, corresponding to a tendency to imitate or avoid the orientation of the interaction partners in the market. Below we show that the noises $\xi(t)$ and $\zeta_{i}(t)$ are necessary to get results comparable to empirical findings on price returns, while the effect of $\eta_{i j}(t)$ is averaged to zero for large $N$. The orientations of all agents are updated synchronously according to the probabilistic rule

$$
\sigma_{i}(t+1)= \begin{cases}1 \quad \text { with probability } p \\ -1 & \text { with probability } 1-p\end{cases}
$$

describing an uncertainty in decision-making, where $p=1 /\left\{1+\exp \left[-2 I_{i}(t)\right]\right\}$ (analogously to the well-known thermal bath dynamics). 
In the following we will be interested in modeling the time series and distributions of the returns $G_{\Delta t}(t)=\ln S(t)-\ln S(t-\Delta t)$, i.e., forward changes in the logarithms of prices $S(t)$ over a time scale $\Delta t$. Relative price changes are proportional to the difference between demand and supply, i.e., between the number of buying and selling decisions. Therefore, after introducing the average orientation $x(t)=N^{-1} \sum_{i=1}^{N} \sigma_{i}(t)$ of agents we get $d S / d t \propto x S$, which after discretizing time yields

$$
G_{\Delta t}(t) \propto \sum_{\tau=0}^{\Delta t-1} x(t-\tau) .
$$

In particular, $G_{1}(t) \propto x(t)$, which enables us to interpret the terms in Eqs. (11) and (21) as follows: Since the average interaction strength $A \xi(t)$ is common to all connections, it is a measure of the average reaction of the agents to the price changes, influencing their decisions via the term $A \xi(t) x(t)$ in the mean field $I_{i}(t)$. The terms $a \eta_{i j}(t)$ describe the fluctuating interaction network while $h \zeta_{i}(t)$ describe the fluctuating environment. The local field in Eq. (11) describes how agent $i$ anticipates price changes by averaging the information on the opinions of his interaction partners and the external information accessible to him.

Before discussing the results of numerical simulations for large $N$, we study the meanfield approximation of our model. For this purpose let us consider the case $A \neq 0, a=0$ and $\zeta_{i}(t)=\zeta(t)$ for all $i$. Then the dynamics of $x(t)$ reduces to a one-dimensional map,

$$
\begin{aligned}
x(t+1) & =\tanh [A \xi(t) x(t)+h \zeta(t)] \\
& \approx A \xi(t) x(t)+h \zeta(t)
\end{aligned}
$$

where the approximate equality holds for $|A \xi(t) x(t)+h \zeta(t)| \ll 1$. The map (5) is a generic model for on-off intermittency if $h=0$ [13], and for attractor bubbling if $h>0$ [14]; let us also note that the approximate linearized form of Eq. (5) belongs to a more general class of stochastic processes with multiplicative noise [9, 12, 16]. In the case $h=0$ and with $\xi(t)$ distributed uniformly in the interval $(-1,1)$, the fixed point $x=0$ of Eq. (5) loses stability for $A \geq \mathrm{e}=2.718 \ldots$, as a result of the so-called blowout bifurcation, while for $h>0$ the stability is lost already for $A \geq 1$ [13,14]. Above the instability threshold the trajectory departs, on average, from zero, but, due to the confining nonlinearity of the map (5), after the departure is randomly reinjected towards $x=0$. Thus the time series of $x(t)$ have intermittent character and consist of laminar phases, during which $x(t) \propto \mathcal{O}(h)$, and chaotic bursts, during which $x(t) \propto \mathcal{O}(1)$. Although the time series for on-off intermitency and 
attractor bubbling look similar, there is a qualitative difference between these phenomena: In the case of on-off intermittency the bursts are caused solely by the multiplicative noise $\xi(t)$ whose amplitude $A$ exceeds the blowout bifurcation threshold, while in the case of attractor bubbling the multiplicative noise only amplifies to macroscopic sizes small deviations from the fixed point caused by the additive noise $\zeta(t)$. In Fig. 1(a), an example of time series $x(t)$ is shown in the attractor bubbling regime. It bears certain resemblance to empirical financial time series of price returns: There are frequent large bursts, corresponding to volatility clustering, separated by long-lasting laminar phases during which the dynamics of the map is governed mainly by the additive noise $h \zeta(t)$.

Another property of the map (5) is that the probability distribution $\rho(x)$ of $x$ (proportional to the probability distribution of $G_{1}$ ) shows pronounced tails obeying power-law scaling. It was shown analytically that for $h=0$ the distribution of $x$ following the linearized multiplicative stochastic process in discrete time (5) obeys the power law scaling $\rho(x) \propto x^{-\alpha-1}$ [16]; this scaling remains valid also for $x \gg h>0$ at least for Gaussian noises $\xi, \eta$ and continuous time [16,17]. Following Ref. [16] the exponent $\alpha$ can be obtained as a non-zero solution of equation $\int_{-\infty}^{\infty} \exp (\alpha y) w(y) d y=A^{\alpha}(1+\alpha)^{-1}=1$, where $y=\ln |A \xi|$ is a random variable with density $w(y)=A^{-1} e^{y}$ in the range $y \leq \ln A$. This means that the cumulative distribution $P(x)$ exhibits power-law tails with $P(x) \propto x^{-\alpha}$. The power law scaling of $\rho(x)$ and the analytic values of $\alpha$ (independent of $h$ ) are confirmed by our numerical simulations (Fig. 1(b)). In order to obtain a value $\alpha>2$ (i.e., within the range observed in empirical stock market time series [3]), the parameter $A$ in Eq. (5) should be in the attractor bubbling regime $1<|A|<\mathrm{e}$, so additive noise with $h \neq 0$ is necessary. Moreover, the cumulative distributions of normalized returns $G_{\Delta t}$ show tails obeying powerlaw scaling with robust exponent $\alpha$ for time scales $\Delta t$ varying over more than one order of magnitude (Fig. 1(c)), a fact also typical of financial time series [3].

It is also known that, close to the blowout bifurcation, the power spectrum of $|x(t)|$ from Eq. (5) shows power law scaling $P(\omega) \propto \omega^{-1 / 2}$ for small $\omega$, suggesting a similar power law scaling of the autocorrelation function of the absolute values of returns. However, in the interesting attractor bubbling regime we observed only slow exponential decay of the autocorrelation of $|x(t)|$.

Now, we turn to numerical multi-agent simulations of the system. Examples of time series of the mean orientation $x(t)$ of agents are shown in Fig. 2(a). It can be seen that, as 
the number of agents increases, laminar phases and chaotic bursts become more distinct, in contrast with many other multi-agent models of stock market [6]. In our model, the laminar phases with $x \approx 0$ correspond to the disordered phase with no preferred orientation, and the bursts with $x \propto \mathcal{O}(1)$ correspond to the ordered phase with a majority of agents sharing one orientation ("herding behavior"). For large $N$, and even for $a>A$, the time series of $x(t)$ resulting from the simulation of Eqs. (1) to (3) and of the mean-field Eq. (5) look similar. Also the scaling exponents $\alpha$ are close to those obtained from the mean field dynamics (5) with the same value of $A$. Note that, in a system with a large but finite number of agents, there are always fluctuations of $x(t)$ around zero in the disordered phase due to the heatbath dynamics of individual agents. These fluctuations play the role of additive noise in Eq. (5), enabling behavior typical of attractor bubbling even if $h=0$ in Eq. (2).

The main outcome of our many-body simulation is that, in a system of many agents, each of which is subject to "thermal bath" fluctuations, attractor bubbling can appear as a collective phenomenon, as a result of time-dependent interactions among agents. The requirement for its appearance is the average interaction strength between agents to vary randomly in time around the value at which the phase transition between the disordered and ordered state occurs. In the thermodynamic limit, the macroscopic system dynamics reduces then to the mean-field dynamics governed by the average interaction strength. The details of the interactions among pairs of agents, and the details of the variation of the mean interaction strength [13], seem unimportant for the qualitative result.

To summarize, in this Letter we generalized a model of social impact to the case of randomly-in-time varying interactions among agents, and we have applied it to simulations of financial time series. Certain empirical properties of these time series, like volatility clustering and a power law distribution of returns, were shown to result from attractor bubbling, which appears in our model in the limit of many interacting agents. Thus we perceive the market as a dynamical system with an extreme kind of intermittency, in contrast with other models with time-dependent interactions between agents [4], which are related rather to self-organized criticality. Due to the lack of topological structure of the interaction network, the mean field approximation of our model quantitatively reflects the multi-agent dynamics. The necessary conditions for the applicability of the model are (i) random fluctuations in time of the average interaction strength between agents (corresponding to their average reaction to price changes), (ii) an uncertainty of decision making analogous to thermal heat 
bath dynamics, and (iii), in the thermodynamic limit, small additive noise simulating the effect of the external environment.

The authors would like to thank the Quandt Foundation of the ALTANA AG for financial support of the project "Nonlinear Dynamics in Models of Complex Systems". This work was supported in part by the Polsih Committee for Scientific Research under grant No. 5 P03B 00721.

Fig. 1 (a) Time series of returns $x(t)$ from the map (可) with $A=2, h=10^{-2}$; (b) Probability distributions $\rho(x) \propto \rho\left(G_{1}\right)$ of returns from the map (司) and fits to the power law scaling $\rho(x) \propto$ $x^{-\alpha-1}$ of their tails for $h=10^{-3}$ and $A=1.6\left(\circ, \alpha=2.67, \alpha_{a n}=2.89\right) ; h=10^{-2}$ and $A=1.6(\square$, $\left.\alpha=2.62, \alpha_{a n}=2.89\right), A=2.0\left(\triangle, \alpha=0.98, \alpha_{a n}=1.0\right), A=2.75\left(\diamond, \alpha=-0.10, \alpha_{a n}=-0.032\right)$; $\alpha_{a n}$ denotes analytic values of $\alpha$. (c) Cumulative distributions of normalized returns $G_{\Delta t}$ (where the angular brackets denote time average) from the map (5) with $A=1.6, h=10^{-2}, \Delta t=1$ (o), $\Delta t=10(\square), \Delta t=100(\triangle), \Delta t=1000(\diamond)$, and comparision with the power law $P\left(G_{\Delta t}\right) \propto G_{\Delta t}^{-\alpha}$ with exponent $\alpha=2.76$. All distributions were obtained from positive returns.

Fig. 2 (a) Time series of returns $x(t)$ from the model with many interacting agents with $A=2.0$, $a=4.0$ and, from above, $N=25$ and $N=4000$; (b) Probability distributions $\rho(x)$ of returns and fits to the power law scaling $\rho(x) \propto x^{-\alpha-1}$ of their tails from the model with $N=1000, h=0$, $a=2 A$, and $A=1.6(\square, \alpha=2.59), A=2.0(\triangle, \alpha=0.98), A=2.75(\diamond, \alpha=-0.13)$. All distributions were obtained from positive returns. 


\section{REFERENCES}

[1] R. N. Mantegna, and H. E. Stanley, An Introduction to Econophysics: Correlations and Complexity in Finance, (Cambridge University Press, Cambridge, UK, 1999); J.P. Bouchaud, and M. Potters, Theory of Financial Risk, (Cambridge University Press, Cambridge, UK, 1999).

[2] R. N. Mantegna, and H. E. Stanley, Nature 376, 46 (1995), Nature 383, 587 (1996); S. Ghashghaie et al., Nature 381, 767 (1996); L. A. Nunes Amaral et al., Phys. Rev. Lett. 80, 1385 (1998); Y. Lee et al., Phys. Rev. Lett. 81, 3275 (1998); N. Vandewalle, and M. Ausloos, Eur. Phys. J. B 4, 257 (1998); J.-P. Bouchaud, and R. Cont, Eur. Phys. J. B 6, 543 (1998); V. Plerou et al., Phys. Rev. Lett. 83, 1471 (1999); L. Laloux et al., Phys. Rev. Lett. 83, 1467 (1999); S. Ciliberti et al., Phys. Rev. Lett. 85, 4848 (2000); J. A. Hołyst, M. Żebrowska, and K. Urbanowicz, Eur. Phys. J. B 20, 531 (2001).

[3] Y. Liu et al., Phys. Rev. E 60, 1390 (1999); P. Gopikrishnan et al., Phys. Rev. E 60, 5305 (1999), Physica A 287, 362 (2000).

[4] R. Cont, and J.-P. Bouchaud, Macroeconom. Dyn. 4, 170 (2000); V. M. Eguíluz, and M. G. Zimmermann, Phys. Rev. Lett. 85, 5659 (2000); I. Chang, and D. Stauffer, Physica A 299, 547 (2001).

[5] T. Lux, and M. Marchesi, Nature 297, 498 (1999).

[6] E. Egenter, T. Lux, and D. Stauffer, Physica A 268, 250 (1999).

[7] M. Youssefmir, and B. Huberman, J. Econ. Behav. Organizat. 32, 101 (1997).

[8] M. Levy, H. Levy, and S. Solomon, Econ. Lett. 45, 103 (1994), J. Phys. I 5, 1087 (1995); M. Levy, S. Solomon, and G. Ram, Int. J. Modern Phys. C 7, 65 (1996).

[9] H. Takayasu, A. Sato, and M. Takayasu, Phys. Rev. Lett. 79, 966 (1997); A. Sato, and H. Takayasu, Physica A 250, 231 (1998).

[10] A. Gaunersdorfer, and C. Hommes, A Nonlinear Structural Model for Volatility Clustering, CeNDEF Working Paper 00-05, University of Amsterdam, http://www.fee.uva.nl/cendef/researchers/hommes/working papers.htm 
[11] J. Cremer, Physica A 246, 377 (1997); G. Iori, Int. J. Modern Phys. C 10, 1149 (1999); D. Chowdhury, and D. Stauffer, Eur. Phys. J. B 8, 477 (1999); T. Kaizoji, Physica A 287, 493 (2000); D. Sornette, Physica A 284, 355 (2000); B. M. Roehner, and D. Sornette, Eur. Phys. J. B 16, 729 (2000); S. Bornholdt, Int. J. Mod. Phys. C 12, 667 (2001).

[12] M. Levy, and S. Solomon, Int. J. Mod. Phys. C 7, 595 (1996); S. Solomon, and M. Levy, Int. J. Mod. Phys. C 7, 745 (1996); D. Sornette, and R. Cont, J. Phys. I 7, 431 (1997).

[13] N. Platt, E. A. Spiegel, and C. Tresser, Phys. Rev. Lett. 70, 279 (1993); J. F. Heagy, N. Platt, and S. M. Hammel, Phys. Rev. E 49, 1140 (1994); E. Ott, and J. C. Sommerer, Phys. Lett. A 188, 39 (1994); A. Krawiecki, and R. A. Kosiński, Int. J. Bifurcation and Chaos Appl. Sci. Eng. 9, 97 (1999).

[14] P. Ashwin, J. Buescu, and I. N. Stewart, Phys. Lett. A 193, 126 (1994); N. Platt, S. M. Hammel, and J. F. Heagy, Phys. Rev. Lett. 72, 3498 (1994); S. C. Venkataramani et al., Phys. Rev. Lett. 77, 5361 (1996).

[15] W. Weidlich, Br. J. Math. Stat. Psychol. 24, 51 (1971), Collective Phenom. 1, 51 (1972), Phys. Rep. 204, 1 (1991); B. Latané, Am. Psychol. 36, 343 (1981); A. Nowak, J. Szamrej, and B. Latané, Psychol. Rev. 97, 362 (1990); M. Lewenstein, A. Nowak, and B. Latané, Phys. Rev. A 45, 763 (1992); D. Helbing, Quantitative Sociodynamics, (Kluwer Academic, Dordrecht, 1995); K. Kacperski, and J. A. Hołyst, J. Stat. Phys. 84, 169 (1996); J. A. Hołyst, K. Kacperski, and F. Schweitzer, Physica A 285, 199 (2000).

[16] Y. Kuramoto, and H. Nakao, Phys. Rev. Lett. 78, 4039 (1997); H. Nakao, Phys. Rev. E 58, 1591 (1998).

[17] S. C. Venkataramani et al., Phys. Lett. A 207, 173 (1995), Physica D 96, 66 (1996); P. Ashwin, and E. Stone, Phys. Rev. E 56, 1635 (1997). 

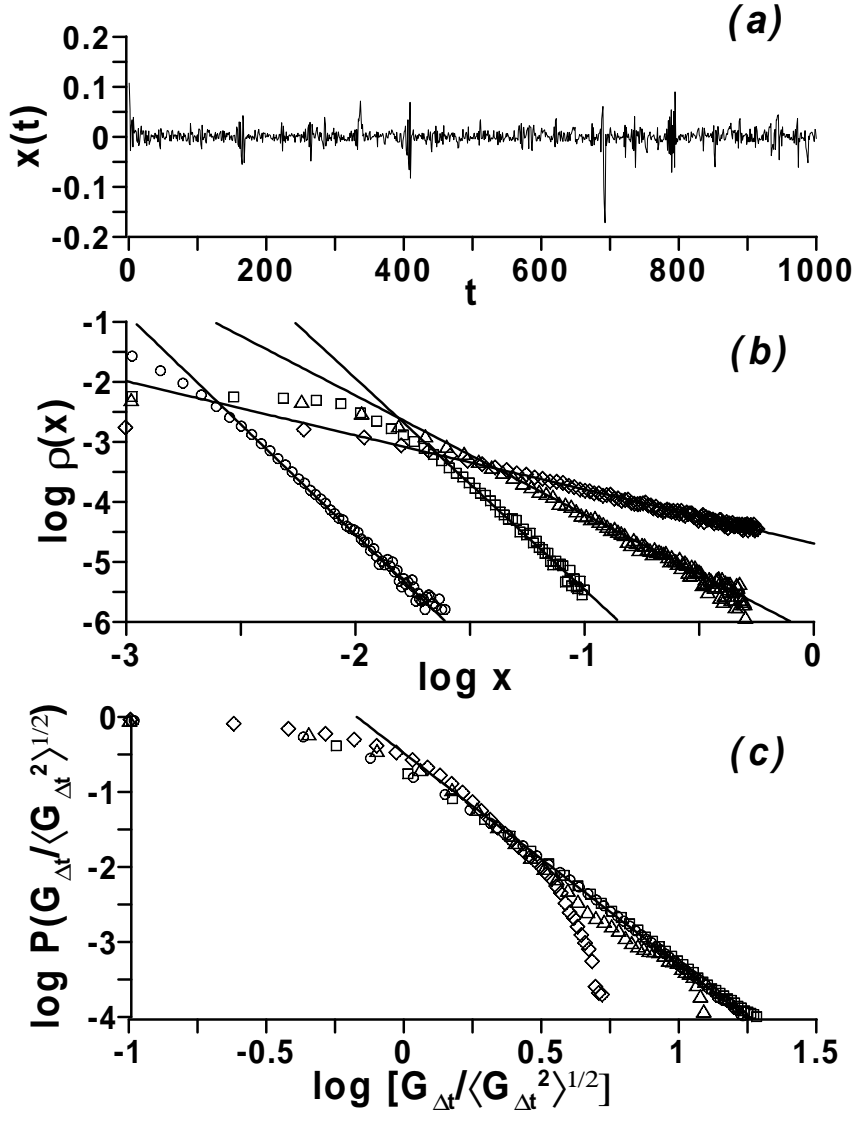

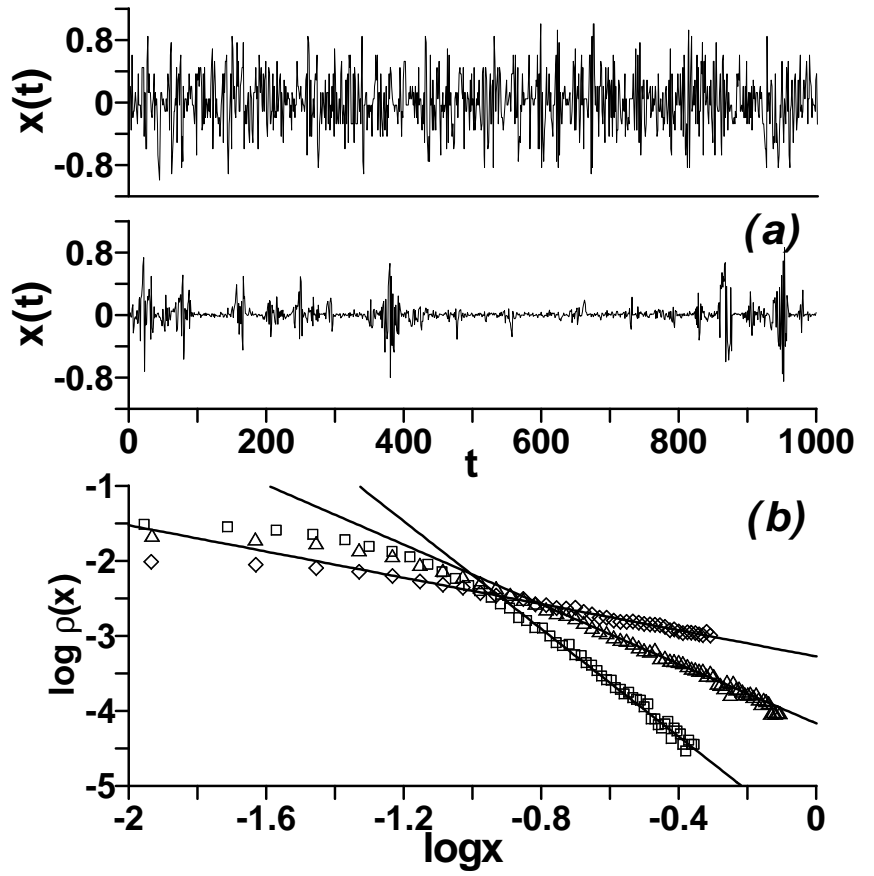